\title{
Auswirkungen der Pandemie: Gesundheitskrise, Ökonomie und Ungleichheit
}

\author{
Mara Linden \\ Department of Human Geography, Goethe University Frankfurt, 60629 Frankfurt am Main, Germany \\ Correspondence: Mara Linden (linden@geo.uni-frankfurt.de)
}

Received: 11 June 2020 - Revised: 2 August 2020 - Accepted: 18 August 2020 - Published: 30 September 2020

Kurzfassung. The focus of this intervention follows the ongoing COVID-19 crisis and its consequences. With both health and the economy as central to this crisis, besides each amplifying the other, in some regards, the two also might stand in conflict. However, both are definitely delivering a number of consequences for humans in different regions and life circumstances across the globe. With the help of several examples, this paper sets out to visualize the unequal distribution of duties, strains, exposure and aftermath of the current health crisis.

\section{Einleitung}

Die aktuelle Pandemie gefährdet die Gesundheit vieler Menschen weltweit, jedoch in unterschiedlichem Maße - z. B. in Bezug darauf, für wen aufgrund von Alter oder Vorerkrankungen das Virus eine größere Gefahr darstellt. Nach bisherigen Zahlen erkranken mehr Männer schwer an Covid-19 als Frauen ${ }^{1}$ (WHO EURO, 2020). Das hat einerseits biologische Gründe, aufgrund hormoneller Unterschiede sowie unterschiedlicher genetischer Reaktionen des Immunsystems, ist aber auch auf vergeschlechtlichte Verhaltensweisen und unterschiedliche Sozialisierung zurückzuführen. Das bedeutet, dass auch Faktoren wie z. B. ökonomischer Status, Herkunft oder Religion Einfluss auf Erkrankungsraten haben (Koerth, 2020).

Die Gesundheitskrise um SARS-CoV-2 wird aber auch tiefgreifende, langanhaltende Folgen für die globale Wirtschaft haben und somit weiterhin Menschen in unterschiedlichen Verhältnissen auf unterschiedliche Weise betreffen, abhängig von z. B. sozioökonomischem Status, race oder location. Globale ökonomische Systeme beruhen notwendigerweise auf sozialen Ungleichheiten und sich daraus ableitenden Hierarchien ebenso wie auf sozial konstruierten Ideen und Diskursen, die diese Ungleichheiten aufgrund von Geschlecht, Nationalität und Herkunft, Alter und anderen Ka- tegorien reproduzieren (Elias, 2011). Gerade diese sehr ungleiche Betroffenheit macht es notwendig, die Geschehnisse aus einer feministischen Perspektive zu analysieren. Eine solche Perspektive soll keineswegs als Wiederbelebung einer ,dual-systems theory“ (Fraser, 2009:98) verstanden werden, die als unterdrückende Achsen Klasse und (das binäre) Geschlecht identifiziert, sondern ist eine stärker intersektional orientierte und globale Verhältnisse zusammendenkende Analysegrundlage. Nur dann haben feministische Ansätze das Potenzial, Lösungen für aktuelle Krisen und deren gesellschaftliche Auswirkungen zu bieten - gerade auch deswegen, weil in Krisenmomenten ethno-kulturelle und biologisch-rassistische Argumente reaktiviert und reproduziert werden, nach denen Minderheiten z. B. für das ,importierte Virus" verantwortlich gemacht werden (Ajayi und Westerveen, 2020). Kritische theoretische Perspektiven können also nicht gender in den Fokus stellen, ohne nicht auch race und location einzubeziehen. Im Folgenden werde ich dazu einige Beispiele ungleicher Verteilung von Aufgaben, Belastung und Folgen der Krise herausgreifen - keinesfalls soll dieser Beitrag aber Anspruch auf eine Vollständigkeit oder des Überblicks erheben. Die genannten Aspekte können bloß Ausschnitte aus verschiedenen Realitäten sein, die situativ und lokal sind.

\footnotetext{
${ }^{1}$ Medizinische Daten beruhen weitestgehend auf einem binären Geschlechtermodell. Es gibt bisher keine Zahlen dazu, in welchem Ausmaß z. B. Trans*-Menschen von Covid-19 betroffen sind.
} 


\section{Gesundheitswesen und Pflege}

Die aktuelle Situation ist eine Krise gesellschaftlicher $\mathrm{Zu}$ sammenhänge, in der teils konfligierende Krisenmomente - primär die der Gesundheitskrise und einer ökonomischen Krise - zusammentreffen und bestehende Ungleichheiten verstärkt werden. Im Globalen Norden wird mit Blick auf das Gesundheitswesen deutlich, dass die Ausbreitung des Coronavirus auf Gesundheitssysteme trifft, die durch eine in den letzten Jahren zugenommene Ökonomisierung der Pflege und des Krankenhauses geschwächt sind. Das im europäischen Vergleich gut aufgestellte deutsche Krankenhauswesen ist vor allem von Personalknappheit, hoher Arbeitsbelastung, Zeitdruck und geringer Anerkennung geprägt, sodass Normalbesetzungen von Schichten nur aus Mindestbesetzungen oder weniger bestehen (Mohan, 2018:198). Regelungen wie die Aussetzung der Pflegepersonaluntergrenzen werden mit Verweis auf die Krise legitimiert. Zugleich werden mit dem Fokus auf medizintechnische Aspekte wie Beatmungsgeräte und Intensivbetten technologische Lösungen hervorgehoben und Pflegepersonal und deren Finanzierung ausgeblendet (Chatzoudis, 2020). Grundsätzlich ist vorhandene Medizintechnik dennoch ein wichtiger Faktor für die Bewältigung der Krise: In Indien beispielsweise gibt es durchschnittlich pro 1000 Einwohner*innen ein halbes Krankenhausbett, was damit laut OECD eine der niedrigsten Raten der Welt ist (Pandey, 2020). In den westafrikanischen Ländern Liberia, Guinea und Sierra Leone, die am stärksten von der Ebola-Epidemie 2014-2016 betroffen waren, werden Vorbereitungen für das Auftreten von CoronaFällen von der wachsenden Sorge begleitet, dass die Auswirkungen der Pandemie hier ebenso verheerend zu spüren sein werden wie Ebola (Mules, 2020). Die WHO warnt, dass gerade einkommensschwache afrikanische Staaten gefährdet sind, da dort u. a. fragile Gesundheitssysteme schon jetzt mit Krankheitsfällen, HIV, Unterernährung und weiteren Gesundheitsproblemen konfrontiert seien (WHO AFRO, 2020). Ungleichheiten bestehen aber auch innerhalb nationaler Gesundheitssysteme: In Brasilien z. B. werden bessergestellte Patient*innen in gut ausgestatteten privaten Krankenhäusern behandelt, während Einwohner*innen einkommensschwächerer Viertel sich auf das schon zuvor überbelastete öffentliche Gesundheitssystem verlassen müssen (Watson, 2020). Fehlende Ausrüstung, mangelndes Personal und geringe Laborkapazitäten für Tests sind dabei nur einige der Aspekte, die nicht nur das Gesundheitssystem schwächen, sondern auch weitreichende Auswirkungen auf das Personal sowie Patient*innen haben.

In der Pflege bestehen dabei grundsätzlich Ungleichheiten durch Beschäftigungsverhältnisse: Im globalen Durchschnitt machen Frauen laut WHO etwa 70 \% der Beschäftigten in Gesundheit und sozialen Bereichen aus, wobei Frauen häufig nur in Teilzeit beschäftigt sind und bedeutend geringer bezahlt werden als ihre männlichen Kollegen (Boniol et al., 2019). Pflegeberufe seien „Frauen- und Teilzeitdomä- nen“, so die deutsche Bundesagentur für Arbeit (2019:8): In Deutschland beträgt der Frauenanteil in der Krankenpflege $80 \%$ und in der Altenpflege sogar $84 \%$, fast die Hälfte aller Arbeitsverhältnisse in Alten- und Krankenpflege sind in Teilzeit (Bundesagentur für Arbeit, 2019:8). Ein hoher Anteil der Beschäftigten hat einen Migrationshintergrund. In einer ausdifferenzierten Betrachtung wird deutlich, dass viele nicht formal qualifiziert und auf Basis kurzer Teilzeitarrangements angestellt sind, und sich damit eine grundsätzlich eher benachteiligte Situation von Pflegekräften mit Migrationshintergrund feststellen lässt (Theobald, 2018). Auch die Unterschiede in der Qualifizierung sind hoch: Während in besser bezahlten Arbeitsplätzen des Gesundheitssektors, z. B. im Berufsfeld der Ärzt*innen, im weltweiten Durchschnitt deutlich weniger Frauen angestellt sind, ist ein überwiegender Teil des - deutlich schlechter bezahlten - Pflegepersonals weiblich (Boniol et al., 2019). Nichtsdestotrotz ist Schutzkleidung meist in Normgrößen verfügbar, die eher auf männlichen Körpern beruhen als auf weiblichen, sodass die Schutzwirkung der Ausrüstung sinkt. Hinzu kommt, dass das Pflegepersonal u. a. Proben nimmt, Patient*innen versorgt und viel näher an Erkrankten ist, und damit meist einem höheren Risiko der Ansteckung ausgesetzt ist als Ärzt*innen (WHO, 2007). In diesem viel direkteren Kontakt mit Infizierten ist also ihr eigenes Infektionsrisiko insgesamt höher (Wenham et al., 2020).

Auch für die Zukunft sieht es nicht gut aus. Die Krisenausrichtung bedeutet, dass andere Bereiche der Gesundheitsversorgung vernachlässigt werden, wie z. B. reproduktive Gesundheitsangebote. So wird in China von überfüllten Krankenhäusern, Abzug des Krankenhauspersonals aus gynäkologischen Bereichen und aufgeschobenen Gesundheitsuntersuchungen von Neugeborenen berichtet (Stevenson, 2020). Auch in den USA trifft die Pandemie auf ein Gesundheitssystem, dessen Müttersterblichkeitsrate seit Jahren weiter steigt, während sie in fast allen anderen Ländern der Welt abnimmt (Kassebaum et al., 2016). Die Sterblichkeitsrate für Women of Colour ist dabei sogar doppelt bis dreifach so hoch. In dieser Situation sind marginalisierte Frauen stärker von den Einschränkungen durch das Coronavirus betroffen, z. B. wenn Krankenhäuser ihre gynäkologischen Abteilungen schließen, Patient*innen nicht mehr aufnehmen oder Besuchsrechte für Familienmitglieder stark einschränken (Guo, 2020). In Texas werden mit Verweis auf die Pandemie die Auseinandersetzungen um das Recht auf körperliche Selbstbestimmung eingeschränkt und Abtreibungen als ,,non-essential“ aufgeschoben (BBC, 2020). Außer Frage steht, dass es sinnvoll ist, die Pflege von Covid-19-Erkrankten zu priorisieren - gleichzeitig muss dabei sichtbar gemacht werden, welche gesundheitlichen Leistungen verschoben und ausgesetzt werden, obgleich sie notwendig oder eigentlich rechtlich abgesichert sind, und welche als weiterhin wichtig eingestuft werden.

Sicher ist, dass sich diese prekären Verhältnisse der Gesundheits- und Pflegebranche in einer Krisensituation noch weiter verschärfen und sich nicht kurzfristig lösen las- 
sen, z. B. durch Anwerbung ausländischer Fachkräfte. Feministische Stimmen kritisieren schon länger, dass der Fachkräftemangel in der Pflege im Globalen Norden nicht mit einer finanziellen und ideellen Aufwertung der Arbeit und Arbeitsverhältnisse einhergehe. Stattdessen führen staatliche Stellen z. B. Initiativen der Arbeitsmigration ein, darunter „Triple Win“2 der Bundesagentur für Arbeit und GIZ. Dass dabei „unerwünschte“ oder weniger gut angesehene Tätigkeiten wie z. B. Pflege an migrantische Fachkräfte ausgelagert werden, ist ein weiterer Aspekt ungleicher hierarchischer Verhältnisse. Und während Deutschland und andere Länder des Globalen Nordens in der komfortablen Situation sind, Kräfte anzuwerben, steht diese Option Ländern des Globalen Südens meist nicht offen - sondern verstärkt eher noch die Entstehung von Pflegelücken im Globalen Süden entlang sogenannter Global Care Chains (Hochschild, 2000).

\section{Arbeitsverhältnisse und Ansteckungsrisiken}

Frauen, die ihre Heimat temporär verlassen, um im Globalen Norden z. B. als Pflegekräfte zu arbeiten, sind darüber hinaus deutlich stärker von Reisebeschränkungen, Veränderungen des Aufenthaltsstatus und anderen die Mobilität betreffenden Maßnahmen betroffen. In Deutschland fehlen mittlerweile viele Pflegerinnen, die sonst aus Polen anreisen, und die nun aufgrund von erschwerten Reisewegen, erhöhter Ansteckungsgefahr unterwegs und polnischen Quarantäneregelungen verständlicherweise den Weg nicht mehr antreten (Zajonz, 2020). Auch Ausgangsbeschränkungen treffen migrantische Arbeitskräfte: In Hong Kong beispielsweise hat die Regierung angeordnet, dass in Haushalten lebende Arbeiter*innen an ihrem einen freien Tag in der Woche das Haus nicht verlassen dürfen. Nicht nur wird ihnen damit der soziale Kontakt nach außen erschwert, sie sind außerdem einem größeren Risiko der Ausbeutung ausgesetzt, weil sie sich den im Haushalt anfallenden Pflichten schwerer entziehen können (Owen, 2020). Verschärfte Arbeitsbedingungen kommen auch in Arbeitsverhältnissen in anderen Kontexten auf, z. B. solche, in denen prekär Beschäftigte genau diejenigen sind, denen keine oder nur geringfügige Möglichkeiten offenstehen, sich vor dem erhöhten Infektionsrisiko ihrer Berufsgruppe zu schützen, darunter Flugbegleiter*innen, Putzkräfte, Kindergärtner*innen, Beschäftigte in Supermärkten und anderen Geschäften, also diverse Berufsgruppen, in denen Menschen ihnen nahe kommen. Zentral ist dabei, dass diese Ungleichheiten und Machtverhältnisse nicht (nur) nach Geschlecht strukturiert sind, sondern genauso durch sozioökonomische Unterschiede, Herkunft und Aufenthaltsstatus geprägt sind.

\footnotetext{
${ }^{2}$ Mit „Triple Win“ sollen qualifizierte Pflegefachkräfte aus Serbien, Bosnien-Herzegowina, von den Philippinen und aus Tunesien für Einrichtungen der Kranken- und Altenpflege gewonnen werden (https://www.arbeitsagentur.de/vor-ort/zav/ Triple-Win-Pflegekraefte, letzter Zugriff: 1. August 2020).
}

Diese strukturellen Bedingungen der Ungleichheit in Arbeitsverhältnissen treffen dabei häufig mit den ökonomischen Realitäten im Haushalt zusammen, in denen Frauen zusätzlich zum Lohnarbeitsverhältnis soziale Reproduktionsarbeit leisten und Haushalt sowie Kinderbetreuung verwalten - soweit ihnen nicht möglich ist, diese als Dienstleistungen auszulagern. Mit dem Abbau des fordistischen Sozialstaats in Europa sind einige der reproduktiven Tätigkeiten zurück in den Haushalt gewandert, wobei hauptsächlich Frauen die Kosten dieser Re-Privatisierung tragen (Pearson und Elson, 2015:24). An anderer Stelle sind Tätigkeiten sozialer Reproduktion in kommerzielle Dienstleistungen gewandelt worden, wobei diese abgewertet, unterbezahlt und somit in großem Umfang von migrantischen, prekarisierten Frauen ausgeführt werden (Young, 2012:46 f.). Somit sind Frauen in den meisten Fällen diejenigen, die innerhalb der Familienverhältnisse die Versorgung von Kranken, Kindern und älteren Menschen übernehmen. In Deutschland, ebenso wie in anderen europäischen Ländern, sind Schulen, Kitas und weitere Angebote der Kinderbetreuung derzeit geschlossen - Frauen sind damit zusätzlich zur Last der Betreuung auch gesundheitlich gefährdet, da nach bisherigem Wissensstand Kinder und Jugendliche kaum Symptome zeigen, den Virus aber dennoch übertragen können. In diesen familiären Betreuungsverhältnissen sind Frauen somit einem größeren Infektionsrisiko ausgesetzt, haben aber kaum Möglichkeiten, diesem auszuweichen. Nach chinesischen Daten ist die Übertragungsrate innerhalb der Familie am höchsten, ähnlich wie zuvor in anderen Gesundheitskrisen wie u. a. Ebola (Gupta, 2020a; WHO, 2007).

\section{Reprivatisierung und Haushalt}

Gerade im Rahmen des Rückzuges des Wohlfahrtsstaates und öffentlicher sozialer Ausgaben im Globalen Norden wird auch von einer Reprivatisierung sozialer Reproduktion und einer ,intensification of exploitation“ (Bakker und Gill, 2003:34) gesprochen. Von dieser Reprivatisierung sind Haushalte stärker betroffen, deren ökonomische Situation grundsätzlich schwächer ist, z. B. alleinerziehende Frauen oder aufgrund von ökonomischen und/oder rassistischen Diskriminierungen betroffene Familien (Young und Schuberth, 2011:94 f.). Steigende Preise und nicht mehr verfügbare, günstige Einkaufswaren sind ein größeres Problem für Menschen mit geringerem Einkommen - nur diejenigen, die es sich leisten können, konnten zu Beginn der Pandemie „Hamsterkäufe“ machen, während alle anderen die für den Alltag notwendigen Dinge nicht mehr erwerben konnten. $\mathrm{Zu}-$ dem fallen Tafeln und öffentliche Nahrungsmittelausgaben weg, was ebenfalls langfristige Auswirkungen auf betroffene Frauen und ihre Familien hat. In Spanien beispielsweise sind davon sowohl Schulkinder als auch Rentner*innen betroffen, die subventioniertes Essen sonst in der Schule bzw. in Sozialzentren erhalten (Negrete, 2020). 
Darüber hinaus sind Haushalte nach wie vor als privat wahrgenommene Orte, in denen Machtdynamiken und Ungleichheitsverhältnisse kaum öffentlich verhandelt werden. Im klassischen ökonomischen Verständnis sind Haushalte als single units oft die kleinste Kategorie der Analyse. Haushalte als Entitäten werden nicht in ihrer genauen Zusammensetzung wahrgenommen und beruhen damit auf einem heteronormativen Ideal. Unterschiedliche Familienverhältnisse, abseits vom Modell einer heterosexuellen Familie mit einem Mann als Kopf und Ernährer der Familie, finden also in klassischen ökonomischen Analysen keinen Platz (Young, 2010:268). Auch Machtbeziehungen innerhalb von Familien bleiben unsichtbar. Besonders gefährlich wird das in der aktuellen Krisensituation, in der durch Ausgangsbeschränkungen und Quarantäne die Zahl der Fälle häuslicher Gewalt drastisch ansteigt. Die WHO stellte schon vor einigen Jahren fest, dass weltweit eine von drei Frauen sexualisierte Gewalt erfährt (WHO, 2018). In Krisenzeiten steige diese Zahl massiv an, was aktuelle Berichte bestätigen. So sei die Anzahl der Hilfegesuche von Personen, die unter Quarantäne Gewalt erfahren, um ein Vielfaches gestiegen - die meisten der Betroffenen sind dabei Frauen, aber auch LGBTQs sind gefährdet (Godin, 2020). In einigen Communities wird die Pandemie gar als Strafe Gottes für Homosexualität, Abtreibung und weitere „Sünden“ gesehen, so z. B. von US-amerikanischen christlichen Extremisten (Ring, 2020). Ein ähnliches Narrativ zirkuliert beispielsweise im Senegal: Nicht nur die Gefahr der Stigmatisierung, vermeintlich an dem Virus Schuld zu sein, bedroht LGBTQs dort, auch die Möglichkeiten, den sie umgebenden unterdrückenden (Familien-)Strukturen auszuweichen, werden durch den Lockdown minimiert (Oudenhuijsen, 2020).

Das, was im (isolierten) Haushalt geschieht - auch Gewalt -, bleibt oft eine „Privatsache“, wobei Heteronormativität, Machtstrukturen und Gewaltverhältnisse unsichtbar bleiben. Dabei ist gerade die Verschränkung dessen, was global geschieht, mit dem, was auf der lokalen und individuellen Ebene passiert und ausgehandelt wird, zentraler Punkt eines globalen kapitalistischen Netzwerks: Einerseits haben Unterbrechungen in lokalen, kleineren Unternehmen Implikationen für das globale Wirtschaftssystem und internationale Handels- und Warenketten - in etwa wie eine Art Domino-Effekt. Andererseits ist der Haushalt als Ort sozialer Reproduktion essentiell für das Fortbestehen gesellschaftlicher Strukturen. Die Thematisierung des privaten und öffentlichen Lebens als politische und ökonomische Grundlage des globalen Systems bleibt in vielen Analysen aus, obwohl gerade häusliche, persönliche und private Tätigkeiten die Aspekte sind, die das ökonomische System stützen. Die hier beschriebenen Ungleichheiten beispielsweise werden im öffentlichen Diskurs wie auch in den meisten ökonomischen Analysen zwar als problematisch anerkannt, doch zu oft nicht als systemisch und strukturell eingeordnet - obwohl sie als solche mit in die politökonomische Analyse der Gesundheitskrise einbezogen werden müssen.

\section{Gesundheit und Ökonomie}

Auch abseits des Gesundheitssystems sind die ökonomischen Folgen der Gesundheitskrise massiv. Diese reichen von Insolvenz und Kurzarbeit anmeldenden Unternehmen bis zu Auswirkungen auf den globalen Finanzmärkten und der Angst vor einer Rezession. Der Generalsekretär der OECD spricht von der Coronakrise als größtem ökonomischen, finanziellen und sozialen Schock des 21. Jahrhunderts, dessen Folgen noch Jahre zu spüren sein werden (Gurría, 2020). In Deutschland ist das Bruttoinlandsprodukt (BIP) im ersten Quartal 2020 um 2,2 \% gesunken (ZeitOnline, 2020). Am Ende des zweiten Quartals ist das BIP im Vergleich zum ersten Quartal um nochmals $10 \%$ gesunken, was ein doppelt so starker Rückgang als während der Finanzkrise 2008/09 ist (Destatis, 2020). Fiskalpakete der EU und EZB sowie die Zinspolitik verschiedener Zentralbanken werden das nicht auffangen können (Irwin, 2020) - auch wenn von der EU nach langen Debatten verabschiedete Finanzhilfen eine Abkehr von der bisherigen Finanzpolitik der EU bedeuten. Unter dem sogenannten EU-Wiederaufbaufonds wird die EUKommission in den Jahren 2020-2024 Anleihen in Höhe von EUR 750 Milliarden aufnehmen, um Kredite an EUMitgliedsstaaten zu vergeben und Ausgaben im Rahmen der europäischen Aufbau- und Resilienzmaßnahmen mitzufinanzieren (Europäischer Rat, 2020). In anderen Regionen der Welt stehen solche Mechanismen jedoch nicht zur Verfügung, was bestehende Differenzen verstärkt und weniger stabile Regionen der Welt noch weiter schwächt. Die Vereinten Nationen warnen eindringlich vor u. a. Nahrungsunsicherheiten in 25 Ländern in Afrika, Lateinamerika und Asien (United Nations, 2020).

Nach Prognosen der International Labour Organisation (ILO) vom März werden die Gesundheitskrise und die damit einhergehenden Einschränkungen weltweit etwa 25 Millionen Arbeitsplätze vernichten (ILO, 2020c). Nicht ganz einen Monat später korrigiert die ILO diese Prognose: Erwartet wird Mitte April, dass die Arbeitslosenzahl im Jahr 2020 signifikant höher sein wird, da derzeit schon etwa $80 \%$ aller Beschäftigten weltweit von partiellen Schließungen ihrer Arbeitsstätten betroffen sind (ILO, 2020a). Teilzeitarbeitsverhältnisse und informelle Jobs sind in Krisenzeiten stärker bedroht, was vor allem die Arbeitsverhältnisse vieler prekär beschäftigter Frauen beeinflusst. In den USA allein sind im April 20.5 Millionen Menschen entlassen worden, von denen der Großteil weiblich und nicht-weiß ist (Gupta, 2020b). Nicht viel anders sieht es im globalen Durchschnitt aus: Die ökonomischen Folgen der Pandemie treffen vor allem jüngere Beschäftigte - und insbesondere junge Frauen -, so Guy Ryder, Generaldirektor der ILO (ILO, 2020b). Gerade der Rückgang von Konsum angesichts der derzeitigen Situation wird einkommensschwächere Frauen stärker treffen, da diese tendenziell in Bereichen der Gastwirtschaft, Einzelhandel und anderen Dienstleistungsbranchen angestellt sind (Owen, 2020). Der Großteil derjenigen, die in unterbezahlten und 
wenig wertgeschätzten Arbeitsplätzen tätig sind, verfügt darüber hinaus nicht über ein finanzielles Polster, das sie im Fall einer plötzlichen Arbeitslosigkeit auffangen kann.

Ein weiterer Punkt ist, dass gesellschaftliche Bereiche quasi hierarchisiert werden, in dem einige als ,systemrelevant" eingestuft werden, während andere scheinbar unwichtiger sind. Das betrifft beispielsweise die Lockerungen in der Pandemie - z. B., dass in Deutschland Geschäfte und Restaurants wieder öffnen, während die Kinderbetreuung für die meisten Familien weiterhin nicht zur Verfügung steht. Diejenigen, die es sich leisten können, Care-Arbeit entlang globaler - oder auch lokaler - Care Chains abzugeben, können die Reprivatisierung damit besser abfangen. Deutlich wird daran zusätzlich, dass Kinder und ihre Interessen unsichtbar bleiben, da sie (noch) keine produktiven Teilnehmer*innen der Gesellschaft sind. Kinderbetreuung als Notfallbetreuung steht, zumindest im deutschen Kontext, nur den in als systemrelevant markierten Branchen arbeitenden Eltern offen oder Kindern, die in ihren Familien von Kindeswohlgefährdung betroffen sind.

Die hier genannten Beispiele sind nur Ausschnitte der unterschiedlichen Auswirkungen der aktuellen Gesundheitskrise auf gesellschaftliche Verhältnisse in verschiedenen Regionen. Noch ist nicht abzusehen, wie sich die aktuelle Situation weiterentwickelt und wie sich das Verhältnis zwischen Gesundheit(skrise), Ökonomie und der darin bestehenden Ungleichheitsverhältnisse verändert. In der Analyse von Gesundheit und Krise sollten langfristige Verschiebungen im Bereich von Gesundheit - Ökonomisierung, Privatisierung wie auch eine Einbindung von Gesundheit in Fragen der nationalen Sicherheit - mit in Analysen der Krise einflieBen, sowohl auf der Skala des Lokalen und Privaten, genauso aber in ihrer Relation zu globalen Verhältnissen und hinsichtlich temporaler und räumlicher Unterschiede. Genauso müssen Geschlechterverhältnisse und weitere Machtstrukturen in preparedness plans und response strategies jetzt und zukünftig aktiv mitgedacht und einbezogen werden. Alternative solidarische Strukturen wie Nachbarschaftsnetzwerke, Ehrenamt und gemeinschaftliche Zusammenarbeit bereitzustellen, wie in verschiedenen deutschen Städten schon geschehen, kann die ungleichen Auswirkungen und Folgen der Pandemie bis zu einem gewissen Grade abmildern - diese solidarischen Strukturen sollten aber keinesfalls in die Falle tappen, die oben genannte Re-Privatisierung zu verstärken und den Staat der Aufgabe zu entheben, ein stabiles, ausfinanziertes Gesundheits- und Pflegesystem bereitzustellen, das über Krisenmomente hinaus geschätzt und finanziell angemessen entlohnt wird.

Datenverfügbarkeit. Für diesen Artikel wurden keine Datensätze genutzt.
Interessenkonflikt. Der Autor erklärt, dass kein Interessenkonflikt besteht.

Begutachtung. This paper was edited by Benedikt Korf and reviewed by one anonymous referee.

\section{Literatur}

Ajayi, F. and Westerveen, L.: COVID-19: Amplifier of racism and inequalities, Commentary, VUB Institute for European Studies, 8 May 2020, available at: https://www.ies.be/node/5484, last access: 4 Jnue 2020.

Bakker, I. and Gill, S. (Eds.): Ontology, Method, and Hypotheses, in: Power, Production and Social Reproduction, in: Human In/security in the Global Political Economy, Palgrave Macmillan, London, 17-41, https://doi.org/10.1057/9780230522404_2, 2003.

BBC: Coronavirus: Texas says abortions ,non-essential“ amid pandemic, BBC News, 24 March 2020, available at: https://www. bbc.com/news/52012243, last access: 29 May 2020.

Boniol, M., McIsaac, M., Xu, L., Wuliji, T, Diallo, K., and Campbell, J.: Gender Equity in the Health Workforce: Analysis of 104 Countries, World Health Organization, Geneve, 2019.

Bundesagentur für Arbeit: Arbeitsmarktsituation im Pflegebereich, online aufrufbar: http://statistik.arbeitsagentur.de (letzter Zugriff: 29. Mai 2020), 2019.

Chatzoudis, G.: Die kapitalistische Produktionsweise ist kein System der Vor- und Fürsorge. Interview mit Robin Mohan über die Ökonomisierung des Gesundheitswesens, L.I.S.A., Gerda-Henkel-Stiftung, 7. April 2020, online aufrufbar: https://lisa.gerda-henkel-stiftung.de/oekonomisierung krankenhaus_mohan, letzter Zugriff: 29. Mai 2020.

Destatis: Bruttoinlandsprodukt im 2. Quartal 2020 um 10,1\% niedriger als im Vorquartal, Pressemitteilung Nr. 287 vom 30. Juli 2020, online aufrufbar: https://www.destatis.de/DE/ Presse/Pressemitteilungen/2020/07/PD20_287_811.html, letzter Zugriff: 2. August 2020.

Elias, J.: Critical Feminist Scholarship and IPE, in: Critical International Political Economy. Dialogue, Debate and Dissensus, edited by: Shields, S., Buff, I., and Macartney, H., Palgrave Macmillan, London, 99-113, https://doi.org/10.1057/9780230299405_7, 2011.

Europäischer Rat: Sondertagung des Europäischen Rates, 17.21. Juli 2020, Europäischer Rat/Rat der Europäischen Union, online aufrufbar: https:/www.consilium.europa.eu/de/meetings/ european-council/2020/07/17-21/, letzter Zugriff: 29. August 2020.

Fraser, N.: Feminism, Capitalism and the Cunning of History, New Left Rev., 56, 97-117, 2009.

Godin, M.: As Cities Around the World Go on Lockdown, Victims of Domestic Violence Look for a Way Out, TIME, 18 March 2020, available at: https://time.com/ 5803887/coronavirus-domestic-violence-victims/, last access: 29 May 2020.

Guo, E.: Coronavirus Threatens an Already Strained Maternal Health System, New York Times, 26 March 2020, available at: https://www.nytimes.com/2020/03/26/us/ 
coronavirus-pregnancy-maternal-health-system.html,

last access: 4 June 2020.

Gupta, A. H.: Why Women May Face a Greater Risk of Catching Coronavirus, New York Times, 12 March 2020, available at: https://www.nytimes.com/2020/03/12/us/ women-coronavirus-greater-risk.html (last access: 4 June 2020), 2020a.

Gupta, A. H.: Why Some Women Call This Recession a ,Shecession', New York Times, 9 May 2020, available at: https://www.nytimes.com/2020/05/09/us/ unemployment-coronavirus-women.html (last access: $3 \mathrm{Ju}$ ne 2020), 2020b.

Gurría, A.: Coronavirus (COVID-19): Joint actions to win the war, Statement of the OECD Secretary-General, 21 March 2020, available at: https://www.oecd.org/about/secretary-general/ Coronavirus-COVID-19-Joint-actions-to-win-the-war.pdf, last access: 29 May 2020.

Hochschild, A. R.: Global Care Chains and Emotional Surplus Value, in: On the Edge. Living with Global Capitalism, edited by: Hutton, W. and Giddens, A., Random House, London, 130-146, 2000 .

ILO: COVID-19 causes devastating losses in working hours and employment, ILO, 7 April 2020, available at: https://www.ilo.org/global/about-the-ilo/newsroom/news/

WCMS_740893/lang--en/index.htm (last access: 29 May 2020), 2020a.

ILO: More than one in six young people out of work due to COVID-19, ILO, 27 May 2020, available at: https://www.ilo.org/global/about-the-ilo/newsroom/news/

WCMS_745879/lang--en/index.htm (last access: 29 May 2020), 2020b.

ILO: Almost 25 million jobs could be lost worldwide as a result of COVID-19, says ILO, 18 March 2020, available at: https://www.ilo.org/global/about-the-ilo/newsroom/news/ WCMS_738742/lang--en/index.htm, (last access: 29 May 2020), 2020c.

Irwin, N.: The Upshot: Why the Outlook for the Economy Just Got Worse, New York Times, 9 March 2020, available at: https://www.nytimes.com/2020/03/09/upshot/ coronavirus-oil-prices-bond-yields-recession.html, last access: 4 June 2020.

Kassebaum, N. J., Steiner, C., Murray, C. J. L., Lopez, A. D., Lozano, R., et al.: Global, regional, and national levels of maternal mortality, 1990-2015: a systematic analysis for the Global Burden of Disease Study 2015, Lancet, 388, 1775-1812, https://doi.org/10.1016/S0140-6736(16)31470-2, 2016, 2016.

Koerth, M.: Why are more men than women dying of Covid-19?, ThirtyFiveEight, 30 April 2020, available at: https://fivethirtyeight.com/features/ why-are-more-men-than-women-dying-of-covid-19/, last access: 29 May 2020.

Mohan, R.: Die Ökonomisierung des Krankenhauses, transcript, Bielefeld, 2018.

Mules, I.: Ebola-hit West African nations on alert amid coronavirus pandemic, DW.com, 18 March 2020, available at: https://p.dw. com/p/3Zf5w, last access: 29 May 2020.

Negrete, C.: Virus und Klassenfrage. Coronavirus in Spanien, Junge Welt, 14. März 2020, online aufrufbar: https://www.jungewelt.de/artikel/374467. coronavirus-in-spanien-virus-und-klassenfrage.html, letzter Zugriff: 29. Mai 2020.

Oudenhuijsen, L.: On being queer and contained. Africa is a Country, 27 April 2020, available at: https: //africasacountry.com/2020/04/on-being-queer-and-contained, last access: 29 May 2020.

Owen, L.: Coronavirus: Five Ways Virus Upheaval Is Hitting Women in Asia, BBC News, 8 March 2020, available at: https://www.bbc.com/news/world-asia-51705199, last access: 29 May 2020.

Pandey, V.: India lockdown: Frontline heroes of the coronavirus war, BBC News, 26 March 2020, available at: https: //www.bbc.com/news/world-asia-india-52039185, last access: 29 May 2020.

Pearson, R. and Elson, D.: Transcending the Impact of the Financial Crisis in the United Kingdom: Towards Plan F - a Feminist Economic Strategy, Feminist Rev., 109, 8-30, https://doi.org/10.1057/fr.2014.42, 2015.

Ring, T.: Coronavirus Is Punishment for ,LGBT Sin“, Says Far-Right Pastor, The Advocate, 6 March 2020, available at: https://www.advocate.com/religion/2020/3/06/ coronavirus-punishment-lgbt-sin-says-far-right-pastor, last access: 29 May 2020.

Stevenson, A.: „I felt like crying“ - Coronavirus Shakes China's Expecting Mothers New York Times, 25 February 2020, available at: https://www.nytimes.com/2020/02/25/business/ coronavirus-china-pregnant.html, last access: 29 May 2020.

Theobald, H.: Pflegearbeit in Deutschland, Japan und Schweden, Hans-Böckler-Stiftung, Düsseldorf, 2018.

United Nations: World's poorest being pushed ,closer to the abyss" of famine, warns WFP chief, UN News, 17 July 2020, available at: https://news.un.org/en/story/2020/07/1068601, last access: 2 August 2020.

Watson, K.: Coronavirus in South America: How it became a class issue, BBC News, 24 March 2020, available at: https://www bbc.com/news/world-latin-america-52023147, last access: $2 \mathrm{Ju}-$ ne 2020.

Wenham, C., Smith, J., and Morgan, R.: Comment COVID-19: The Gendered Impacts of the Outbreak, Lancet, 395, 846-848, https://doi.org/10.1016/S0140-6736(20)30526-2, 2020.

WHO: Addressing Sex and Gender in Epidemic-Prone Infectious Diseases, World Health Organization, Geneve, 2007.

WHO: Violence against women. Strengthening the health response in times of crisis, World Health Organization, available at: https://www.who.int/news-room/feature-stories/detail/ violence-against-women (last access: 29 May 2020), 2018.

WHO AFRO: COVID-19 Situation Update for the WHO African Region, External Situation Report 4, World Health Organization, available at: https://apps.who.int/iris/bitstream/handle/10665/ 331587/SITREP_COVID-19_WHOAFRO_20200325-eng.pdf, last access: 29 May 2020.

WHO EURO: COVID-19 weekly surveillance report. Data for the week of 4-10 May 2002 (Epi week 19), World Health Organization, available at: http://www.euro.who.int/ en/health-topics/health-emergencies/coronavirus-covid-19/ weekly-surveillance-report, last access: 29 May 2020.

Young, B.: The gendered dimensions of Money, Finance, and the Subprime Crisis, in: Gender and Economics. Feministische Kritik der politischen Ökonomie, Herausgeber: Bauhardt, C. und 
Gülay, Ç., VS Verlag für Sozialwissenschaften, Wiesbaden, 258277, 2010.

Young, B.: Zwei Getrennte Welten? Finanzökonomie und Geschlechterforschung, in: Macht oder ökonomisches Gesetz. Zum Zusammenhang von Krise und Geschlecht, Herausgeber: KurzScherf, I. und Scheele, A., Westfälisches Dampfboot, Münster, 36-51, 2012.

Young, B. und Schuberth, H.: Der globale Finanz-Crash und seine Bedeutung für Gender, in: Perspektiven auf die Finanzkrise, Herausgeber: Scherrer, C., Dürmeier, T., und Overwien, B., Verlag Barbara Budrich, Opladen, 89-105, 2011.
Zajonz, D.: Polnische Pflegerinnen bleiben zu Hause, tagesschau.de, 24. März 2020, online aufrufbar: https://www. tagesschau.de/inland/corona-pflegekraefte-polen-101.html, letzter Zugriff: 29. Mai 2020.

ZeitOnline: Deutschlands Wirtschaft stürzt in Rezession, ZeitOnline, 15. Mai 2020, online aufrufbar: https://www.zeit.de/wirtschaft/2020-05/statistisches-bundesamtbip-sinkt-im-ersten-quartal-um-2-2-, letzter Zugriff: 29. Mai 2020. 